\title{
Electron microscopy combined with spatial analysis: quantitative mapping of the nano-assemblies of plasma membrane-associating proteins and lipids
}

\author{
Yong Zhou $^{1 \bowtie}$, John F. Hancock ${ }^{1 \bowtie}$ \\ 1 Department of Integrative Biology and Pharmacology, The University of Texas Health Science Center at Houston, \\ Houston, TX 77030, USA
}

Received: 18 March 2017 / Accepted: 10 February 2018 / Published online: 25 July 2018

\begin{abstract}
The plasma membrane (PM) is a complex environment consisting of $>700$ species of lipids and many different types of membrane-associating proteins. These lipids and membrane proteins are distributed heterogeneously into nanometer-sized domains, called nanoclusters. The lateral spatial segregation in the PM gives rise to different curvature and lipid composition, which determines the efficiency of effector binding and signal transmission. Here, we describe an electron microscopy (EM)-spatial mapping technique to quantify the extent of nanoclusters formation in the PM. The nano-assemblies in the PM are quantified via expressing the GFP-tagged proteins or lipid-binding domains in the cells, which are then immunolabeled with the gold nanoparticles pre-coupled to the anti-GFP antibody. The gold nanoparticles are visualized via the transmission EM at high magnification. The statistical analysis of the Ripley's $K$-function calculates the spatial distribution of the gold nanoparticles. Important spatial parameters, such as the extent of nanoclustering, the clustered fraction, the number of proteins per cluster, the optimal size of a nanocluster, and the number of proteins localized to the PM, can be calculated. Further detailed aggregation pattern, such as the populations of monomers, dimers, trimers, and higher ordered oligomers, can also be extracted from the spatial analysis. The EM-bivariate analysis quantifies the extent of co-localization between two different components in the PM and provides key information on the protein-protein and the protein-lipid interactions over a long-distance scale from 8 to $240 \mathrm{~nm}$.
\end{abstract}

Keywords Electron microscopy, Spatial distribution, Lipid-anchored proteins, Nanoclustering, Signal transduction, Ripley's $K$-function

\section{INTRODUCTION}

The plasma membrane (PM) is a highly complex nanoand micro-environment with $\sim 700$ different species of lipids and many membrane-associating proteins (Simons and Toomre 2000). Biophysical studies using synthetic model bilayers, isolated native giant plasma membrane vesicles (GPMVs) and intact PM sheets, fixed

$\bowtie$ Correspondence: Yong.Zhou@uth.tmc.edu (Y. Zhou), John.F.Hancock@uth.tmc.edu (J. F. Hancock) cells and intact tissues all demonstrate that the lipids and the proteins laterally segregate into nanoclusters with diameters from $<20 \mathrm{~nm}$ to a few hundred nanometers (Baumgart et al. 2003; Levental et al. 2011; Plowman et al. 2005; Prior et al. 2003a; Veatch and Keller 2002). This kind of nanoclustering gives rise to a vastly complex environment in the PM with each membrane protein possessing distinct lipid environment close to its vicinity (Zhou et al. 2014, 2015, 2017). The purpose of such complex lateral spatial segregation is still not clear. However, as many downstream effectors contain the specific lipid-binding domains, such as 
the $\mathrm{C} 2$ and the $\mathrm{PH}$ domains, it is apparent that the PM nanoclustering, along with the distinct lipid composition, is critical to effector recruitment and signal transmission (Zhou et al. 2015, 2017).

It is difficult to characterize the lateral spatial distribution of the lipids and the membrane proteins. We utilized a quantitative imaging technique of electron microscopy (EM) combined with spatial mapping to mathematically determine the extent of nanoclustering of the lipids or the proteins in the PM (Prior et al. $2003 a, b)$. To determine the univariate nanoclustering of a membrane protein, we ectopically expressed the GFPtagged protein of interest in the cells and attach the intact PM sheets to the copper EM grids coated with pioloform and poly-L-lysine. The location of the GFPtagged protein was then marked by incubating the PM sheets with a solution containing 4.5-nm gold nanoparticles pre-coupled to anti-GFP antibody. The gold particle distribution was then imaged and digitized using the transmission EM. The coordinates of the gold particles were assigned using ImageJ, and the extent of nanoclustering of the gold particles was calculated using the statistical analysis of Ripley's $K$-function. To quantify the univariate nanoclustering of a PM lipid, we expressed a GFP-tagged specific lipid-binding motif (Zhou et al. 2014, 2015, 2017). For example, we expressed GFP-LactC2 to label the location of phosphatidylserine (PS) in the PM since the C2 domain specifically binds to PS (Zhou et al. 2014, 2015, 2017). The location and the spatial distribution of PS can then be visualized with the gold particle labeling and the Ripley's $K$-function analysis. The same technique can also be applied to study the co-localization between two distinct components in the PM (Zhou et al. 2014, 2015, 2017). We expressed one component tagged with GFP and another tagged with RFP and label the components with 6-nm gold particles coupled to antiGFP and 2-nm gold particles linked to anti-RFP (Prior et al. 2003a; Zhou et al. 2012, 2014, 2015, 2017). The co-localization between the larger 6-nm gold and the smaller 2-nm gold can be calculated using the bivariate $K$-function. Using the bivariate technique, we can map the detailed protein-protein and protein-lipid spatial nano-assembly in the PM. Combining the univariate and the bivariate analyses, we can deduce the complex spatial environment in the PM: how the extent of nanoclustering of a certain membrane protein along with its complex lipid composition influence the downstream effector binding and the signaling activities (Zhou et al. 2014, 2015, 2017).

\section{PROTOCOL}

\section{Preparation of reagents for EM gold-labeling}

\section{Solutions}

(1) $1 \%$ trisodium citrate: $0.5 \mathrm{~g}$ of sodium citrate dissolved in $50 \mathrm{ml}$ of deionized water, stored at room temperature.

(2) $1 \%$ tannic acid: 0.5 g Aleppo tannin dissolved in $50 \mathrm{ml}$ of deionized water, stored at room temperature.

(3) $25 \mathrm{mmol} / \mathrm{L}$ potassium carbonate: $0.173 \mathrm{~g}$ of potassium carbonate dissolved in deionized water, stored at room temperature.

(4) Reducing solution: $1 \%$ trisodium citrate, $1 \%$ tannic acid, $25 \mathrm{mmol} / \mathrm{L}$ potassium carbonate and deionized water for a final volume of $10 \mathrm{ml}$. The amount of trisodium citrate, tannic acid, and potassium carbonate varies for different-sized gold particles (see Table 1).

(5) 1\% gold chloride (stock): $1 \mathrm{~g}$ of gold chloride dissolved in $100 \mathrm{ml}$ deionized water, stored at $4{ }^{\circ} \mathrm{C}$ for $>2$ years.

(6) Gold solution (for making the gold nanoparticles): $0.5 \mathrm{ml}$ of $1 \%$ gold chloride dissolved in deionized water.

(7) 10\% BSA: 2 g of BSA dissolved in $20 \mathrm{ml}$ of PBS, stored at $4{ }^{\circ} \mathrm{C}$.

(8) $0.1 \%$ BSA in PBS: $0.5 \mathrm{ml}$ of $10 \%$ BSA diluted in $50 \mathrm{ml}$ PBS.

(9) Glycerol gradient (10\%-40\%): varying amount of glycerol and $50 \mu \mathrm{l}$ BSA dissolved in PBS for a final volume of $5 \mathrm{ml}$ (see Table 2).

(10) $2 \%$ methyl cellulose: $2 \mathrm{~g}$ of methyl cellulose dissolved in $98 \mathrm{ml}$ of deionized water, stored at $4{ }^{\circ} \mathrm{C}$.

(11) $10 \times$ KOAc buffer (stock solution): 11.92 g HEPES, $22.58 \mathrm{~g}$ potassium acetate, and $1.02 \mathrm{~g}$ magnesium chloride dissolved in $180 \mathrm{ml}$ deionized water,

Table 1 The amount of tannic acid and potassium carbonate for different-sized gold particles

\begin{tabular}{lllllll}
\hline & \multicolumn{6}{c}{ Gold nanoparticle size (nm) } \\
\cline { 2 - 7 } & $2-3$ & 4 & 4.5 & 5.5 & 6 & 7.5 \\
\hline $1 \%$ tannic acid $(\mathrm{ml})$ & 2.5 & 1.25 & 0.75 & 0.5 & 0.25 & 0.13 \\
$25 \mathrm{mmol} / \mathrm{l} \mathrm{K}_{2} \mathrm{CO}_{3}(\mathrm{ml})$ & 2.5 & 1.25 & 0.75 & 0.5 & 0.25 & 0.13 \\
\hline
\end{tabular}


Table 2 Varying amount of glycerol and 10\% BSA dissolved in PBS for a final volume of $5 \mathrm{ml}$

\begin{tabular}{llllllll}
\hline & $\%$ Glycerol & & & & & \\
\cline { 2 - 7 } & 10 & 15 & 20 & 25 & 30 & 35 & 40 \\
\hline Glycerol (ml) & 0.5 & 0.75 & 1 & 1.25 & 1.5 & 1.75 & 2 \\
$10 \%$ BSA (ml) & 0.05 & 0.05 & 0.05 & 0.05 & 0.05 & 0.05 & 0.05 \\
PBS (ml) & 4.45 & 4.2 & 3.95 & 3.7 & 3.45 & 3.2 & 2.95 \\
\hline
\end{tabular}

stock stored at $-20{ }^{\circ} \mathrm{C}$ while the working solution stored at $4{ }^{\circ} \mathrm{C}$.

(12) Fixative: $2 \mathrm{ml}$ of $16 \%$ paraformaldehyde, $32 \mu \mathrm{l}$ of $25 \%$ glutaraldehyde, and $0.8 \mathrm{ml}$ of $10 \times \mathrm{KOAc}$ stock combined with $5.17 \mathrm{ml}$ of deionized water, stored at $4{ }^{\circ} \mathrm{C}$.

(13) $25 \mathrm{mmol} / \mathrm{L}$ glycine: $93.8 \mathrm{mg}$ glycine dissolved in $50 \mathrm{ml}$ of PBS. Aliquots of $1 \mathrm{ml}$ stored at $4{ }^{\circ} \mathrm{C}$.

(14) $10 \%$ fish skin gelatin: $3 \mathrm{ml}$ of $45 \%$ fish skin gelatin solution combined with $11.5 \mathrm{ml}$ of PBS and stored at $4{ }^{\circ} \mathrm{C}$.

(15) Blocking solution: $100 \mu \mathrm{l}$ of $10 \%$ fish skin gelatin and $100 \mu \mathrm{l}$ of $10 \%$ BSA combined with $9.6 \mathrm{ml}$ of $1 \times$ KOAc buffer (diluted from the $10 \times$ stock).

(16) 3\% uranyl acetate: $1.5 \mathrm{~g}$ of uranyl acetate dissolved in $50 \mathrm{ml}$ of deionized water and stored at $4{ }^{\circ} \mathrm{C}$.

\section{Preparation of antibody coupled gold nanoparticles}

(1) Add $10 \mathrm{ml}$ of reducing solution (see section "Solutions" (4)) and $40 \mathrm{ml}$ of gold solution (see section "Solutions" (6)) in separate flasks and heat to $\sim 60{ }^{\circ} \mathrm{C}$. The amount of solutions added differs based on the size of the gold particles (see Table 1). We typically make 4.5 -nm gold particles.

(2) Combine two solutions once temperature reaches $60{ }^{\circ} \mathrm{C}$.

(3) Boil the combined solution for 5 min with constant stirring. Proper colloid formation should yield a maroon color solution.

(4) Cool down the solution by leaving the flask on ice.

(5) Adjust the $\mathrm{pH}$ to $\sim 8.5$ using $\mathrm{pH}$ paper only (gold solution can damage $\mathrm{pH}$ electrodes). We typically use $0.5 \mathrm{~mol} / \mathrm{L} \mathrm{NaOH}$ solution to adjust the $\mathrm{pH}$. First add $\sim 150 \mu \mathrm{l}$ and then $10 \mu \mathrm{l}$ increments.

(6) To determine the amount of anti-GFP antibody needed, we add 0-5 $\mu \mathrm{g}$ of anti-GFP antibody (diluted to $20 \mu \mathrm{l}$ final volume using deionized water) into $250 \mu \mathrm{l}$ of gold solution to titrate the optimal antibody level.

(7) Incubate the mix at room temperature for $5 \mathrm{~min}$.

(A) Add $100 \mu \mathrm{l}$ of $10 \% \mathrm{NaCl}$ and mix well.

(B) Solutions become bluer in color as more antibodies are present. The optimal amount of the antibody (the lowest amount of antibody) is indicated by the tube that no longer changes its color to blue.

(8) Calculate the amount antibody needed for the total amount of gold solution available and combine antibody with the gold solution.

(9) Incubate the mix for $10 \mathrm{~min}$ at room temperature and add 10\% BSA to the gold/antibody solution to achieve a final BSA concentration of $0.1 \%$.

(10) Centrifuge the mixed gold-antibody solution at $100,000 \mathrm{~g}$ (for $4-6 \mathrm{~nm}$ gold) or $120,000 \mathrm{~g}$ (for 2-3 nm gold) for $1 \mathrm{~h}$ at $4{ }^{\circ} \mathrm{C}$.

(11) Collect the gold-antibody complex by aspirating the loose pallet using a micropipettor. Note that the solid round pellet on the side of tube is the unbound gold and should be discarded.

(12) For univariate analysis, the gold-antibody complex made here should be appropriate to use. For bivariate analysis, the requirement for accurate gold particle size is stricter. The gold particles made using above procedure should be further processed.

(13) To further process, load the gold-antibody complex obtained from above procedure into $5 \mathrm{ml}$ ultracentrifuge tubes containing various amount of glycerol (see Table 2).

(14) Centrifuge $100,000 \mathrm{~g}$ for $6 \mathrm{~nm}$ gold or $120,000 \mathrm{~g}$ for 2-3 nm gold for $1 \mathrm{~h}$ at $4{ }^{\circ} \mathrm{C}$.

(15) Collect gold solution in the top half of the gradient.

(16) To calibrate the gold size distribution, pipette $100 \mu \mathrm{l}$ of the gold solution onto a parafilm sheet and place a pioloform- and poly-L-lysine-coated EM copper grid on the bubble. Incubate $5 \mathrm{~min}$ and let dry for at least $30 \mathrm{~min}$ before EM imaging.

\section{Pioloform- and poly-L-lysine coating EM grids}

\section{Pioloform-coating}

(1) Dissolve $0.4 \mathrm{~g}$ of pioloform in $50 \mathrm{ml}$ of chloroform and shake well to mix. 
(2) Fill a 25-ml cylindrical separatory funnel half-full with the pioloform/chloroform solution and dip an ethanol-cleaned glass slide into the funnel.

(3) Drain the pioloform/chloroform solution, leaving a thin film of pioloform on the slide.

(4) Use a razor blade to cut lines along the edges of the pioloform film on the glass slide.

(5) Slowly dip the glass slide into deionized water in a small bowl. If the pioloform film has been properly formed and cut, the film will slide off the glass slide and float on the water surface.

(6) Carefully position copper or gold EM grids onto the pioloform film with the dull side down. Approximately $40-50$ grids can be placed on a pioloform film.

(7) Place a clean glass slide coated with a self-adhesive paper vertically above the pioloform film and slowly pushing down, allowing the film to coat the glass slide. Pioloform-coated grids are then left to dry overnight.

\section{Poly-L-lysine coating}

(1) Pipette a 2-ml poly-L-lysine bubble on a sheet of parafilm. Slowly cut the pioloform-coated EM grids using fine-tipped tweezers and place on the poly-Llysine bubble with the dull side (pioloform-coated) down.

(2) Allow incubation for $5 \mathrm{~min}$ before washing the grids twice by placing the grids on a 2 -ml deionized water bubble.

(3) The poly-L-lysine-coated grids are then left to dry overnight.

\section{PM rip-off}

PM rip-off can be performed to attach either apical PM or basolateral PM onto the grids. Copper grids are used for apical PM rip-off, while gold grids are used for basolateral PM attachment since copper is toxic to cells. The initial steps differ slightly for the two techniques.

\section{Apical PM rip-off}

(1) Cells grown on glass coverslips in a 3.5-cm dish are washed twice with PBS. The glass coverslip with the appropriate cell confluency $(80 \%-90 \%)$ is then placed on a piece of clean filter paper with the cell side facing up. Two pioloform- and poly-L-lysinecoated copper EM grids are then positioned on cells with the dull side down (with the pioloform- and poly-L-lysine-coated side facing the cells on the glass coverslips).

(2) A rubber bung is used to press down on the EM grids to attach the apical PM to the grids.

(3) Approximately $100 \mu \mathrm{l} 1 \times$ KOAc solution is pipetted onto the glass coverslip around the copper EM grids, making sure not to cover the grids with KOAc solution. The surface tension of the aqueous solution will pop the grids off the glass coverslip, with only the intact apical PM sheets firmly attached to the grids and leaving the rest of the cells on the glass coverslips.

\section{Basolateral PM rip-off}

(1) When the basolateral PM needs to be collected, cells are seeded directly over the pioloform- and poly-L-lysine-coated gold EM grids.

(2) At the time of experiment, the gold EM grids are washed twice with PBS and placed on a piece of clean filter paper. Another piece of clean filter paper pre-wetted with PBS solution is then placed on the top for $10 \mathrm{~min}$.

(3) The wet filter paper on the top is slowly peeled off, leaving only the basal PM attached to the gold EM grids.

(4) To further eliminate endomembranes, a PBSwetted glass coverslip is placed on top of the gold EM grids.

(5) A rubber bung is used to press down on the coverslip. A $100 \mu \mathrm{l} 1 \times$ KOAc bubble is pipetted onto the glass coverslip around the gold EM grids, making sure not to cover the grids with KOAc solution. The surface tension of the aqueous solution will pop the grids off the glass coverslip, with only the basal PM sheets firmly attached to the grids and leaving the rest of the cells on the glass coverslips.

\section{Fixing and labeling procedure}

(1) EM grids with flattened PM are then placed on a $50-\mu$ fixative bubble for $10 \mathrm{~min}$. Note that, the protocol from this point on is the same for both apical and basolateral PM rip-off.

(2) After blotting the EM grids by position the grids on a piece of filter paper at a $45^{\circ}$ angle, the grids are then moved to a $100 \mu \mathrm{l}$ PBS bubble for $5 \mathrm{~min}$.

(3) EM grids are quenched by three consecutive $100 \mu \mathrm{l}$ quenching bubbles $(1 \times$ glycine solution diluted in $1 \times \mathrm{KOAc})$. 
(4) EM grids are then placed on a $100 \mu \mathrm{l}$ blocking solution bubble for $20 \mathrm{~min}$.

(5) After blotting, the grids are then placed on a $10-\mu \mathrm{l}$ bubble containing gold nanoparticles coupled to anti-GFP antibody for $30 \mathrm{~min}$. For bivariate experiments, the grids should be incubated with large 6-nm gold coupled to anti-GFP first, blocked for another $5 \mathrm{~min}$, then incubated with 2-nm small gold coupled to anti-RFP antibody.

(6) After blotting, the grids are placed on five consecutive $100 \mu \mathrm{l}$ blocking solution bubbles, each for $5 \mathrm{~min}$

(7) After blotting, the grids are then placed on five consecutive $100 \mu \mathrm{l}$ deionized water bubbles. Make sure to wash the tweezers with deionized water thoroughly between each step.

(8) Move grids onto a $100 \mu \mathrm{l}$ uranyl acetate bubble (10:1 dilution using methyl cellulose) on ice for $10 \mathrm{~min}$.

(9) Scoop the grids from uranyl acetate bubbles using a grid loop and blot off the excess uranyl acetate on a piece of filter paper. Allow grids to dry overnight.

\section{Imaging and analysis}

\section{Obtain and digitize images}

Obtain and digitize images of PM sheets with proper gold labeling using a transmission EM at the magnification of $100,000 \times$ (Fig. 1A).

\section{Data analysis}

(1) Univariate $K$-function: the spatial distribution of a single species labeled with $4.5-\mathrm{nm}$ gold coupled to anti-GFP.

(A) Open an EM image file in Image J and assign the $x$ and $y$ coordinates to each gold particle within a selected $1 \mu \mathrm{m}^{2}$ area (Fig. 1B). Ensure that the gold particles in the selected area distribute in the most uniform manner, where no visible pattern can be discerned by naked eye.

i. The raw EM images saved as TIFF file is opened in the Image J.

ii. A $1 \mu \mathrm{m}^{2}$ PM sheet area is selected and cropped, which is further smoothened.

iii. The background of the image is subtracted using a rolling ball radius of six pixels.

iv. The gold nanoparticles are then highlighted using the Threshold function.

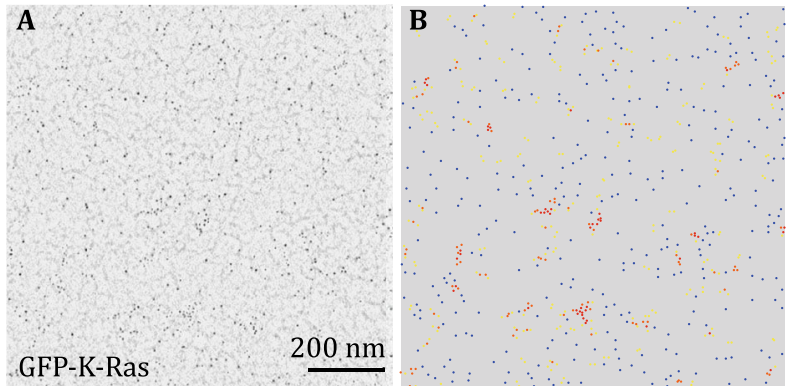

C

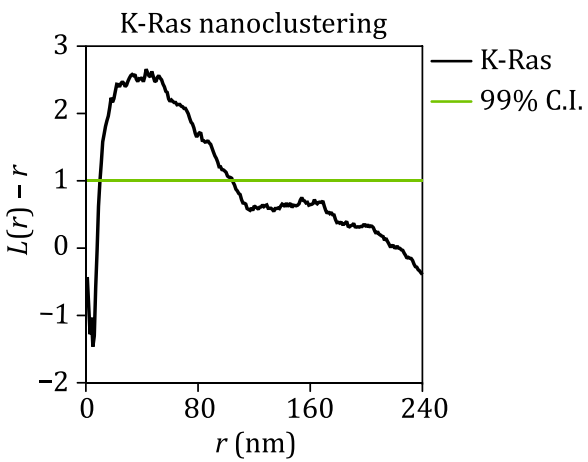

Fig. 1 Univariate nanoclustering analysis quantifies the lateral spatial distribution of a single population of gold nanoparticles on the intact plasma membrane sheets. A An intact PM sheet of kidney cells ectopically expressing GFP-K-Ras was attached to an EM grid, fixed and immunolabeled with $4.5-\mathrm{nm}$ gold nanoparticles. Gold distribution on the intact PM sheet was imaged using transmission EM at 100,000 $\times$ magnification. B $x$ and $y$-coordinates of the gold particles were assigned using ImageJ and were used to calculate the spatial distribution. Blue dots indicate monomers; yellow dots indicate dimers; orange dots indicate trimers; red dots indicate higher ordered oligomers. C The extent of nanoclustering, $L(r)-r$, was plotted against the length scale, $r$, in nanometer. $L(r)-r$ values above the $99 \%$ confidence interval $(99 \% \mathrm{CI})$ indicate statistically meaningful clustering

v. The $x$ and $y$ coordinates to each gold particle are assigned using the "Analyze particle" function, with particle size range of 2-80 pixel $^{2}$.

(B) The clustering of gold particles is analyzed using variations of the statistical analysis of Ripley's K-function (Ripley 1977) (Eqs. 1-2):

$$
\begin{aligned}
& K(r)=A n^{-2} \sum_{i \neq j} w_{i j} 1\left(\left\|x_{i}-x_{j}\right\| \leq r\right) \\
& L(r)-r=\sqrt{\frac{K(r)}{\pi}}-r
\end{aligned}
$$

where $K(r)$ describes the univariate spatial $K$ function for the gold nanoparticles within a selected PM area defined as $A ; n$ is the total number of gold particles within the PM area; $r$ is the length scale originating from each gold particle; || || indicates the Euclidean distance, 
with 1( . ) given a defined value of 1 if $\left\|x_{i}-x_{j}\right\| \leq r$ and a value of 0 if $\left\|x_{i}-x_{j}\right\|>r$; and $w_{i j}^{-1}$ is the fraction of the circumference of a circle with center at $x_{i}$ and a radius $\left\|x_{i}-x_{j}\right\| . K$-function is then standardized to yield $L(r)-r$, via a $99 \%$ confidence interval (CI) estimated by Monte Carlo simulations (Fig. 1C). Typically, a minimum of 15-20 PM sheets are imaged and analyzed for each condition.

(C) The statistical significance of differences between replicated point patterns is evaluated in bootstrap tests constructed as described (Diggle et al. 2000; Plowman et al. 2005).

(D) The number of gold nanoparticles is also counted within the same $1 \mu \mathrm{m}^{2} \mathrm{PM}$ area used for the clustering analysis above. The gold number is an estimate of the level of the targeted membrane proteins on the PM.

i. To ensure that the changing immunogold-labeling reflects a changing protein level, but not changing total expression level of the protein, Western blotting using anti-GFP antibody is usually run to validate the total expression level.

ii. The statistical significance between the comparing sets is evaluated using the one-way ANOVA.

(2) Bivariate $K$-function: co-localization of two populations of proteins/lipid-biding domains labeled by different-sized gold particles (Fig. 2A, 6-nm gold coupled to anti-GFP and 2-nm gold linked to antiRFP) (Prior et al. 2003b; Zhou et al. 2012, 2014).

(A) Open an EM image in ImageJ, assign the $x$ and $y$ coordinates of each small and large gold particle within a selected $1 \mu \mathrm{m}^{2}$ area, and save them in separate files (Fig. 2B).

(B) How each big gold particle $(6 \mathrm{~nm})$ co-clusters with all small particles $(2 \mathrm{~nm})$ and how each small gold co-localizes with all big gold particles are analyzed using a variation of the Ripley's $K$-function, bivariate $K$-functions (Eqs. 3-6):

$$
K_{\mathrm{biv}}(r)=\left(n_{\mathrm{b}}+n_{\mathrm{s}}\right)^{-1}\left[n_{\mathrm{b}} K_{\mathrm{sb}}(r)+n_{\mathrm{s}} K_{\mathrm{bs}}(r)\right]
$$
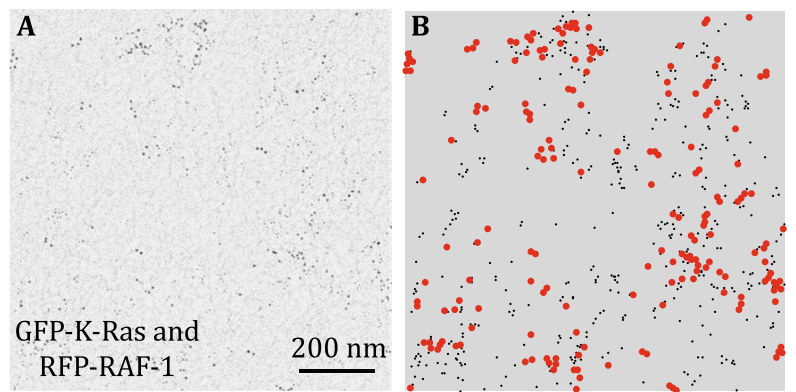

C

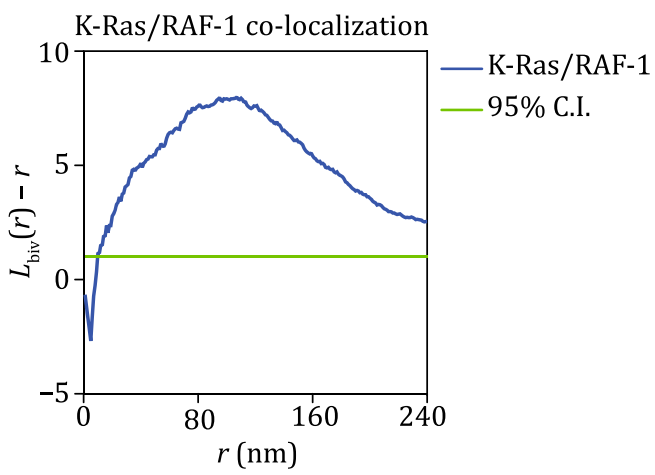

Fig. 2 Bivariate co-clustering analysis characterizes the co-localization between two populations of gold nanoparticles. A An intact PM sheet of kidney cells ectopically co-expressing GFP-K-Ras and RFPRAF-1 was attached to an EM grid, fixed and immunolabeled with 6-nm gold labeling GFP and 2-nm gold labeling RFP. Gold distribution on the intact PM sheet was imaged using transmission EM at 100,000 $\times$ magnification. B $x$ and $y$-coordinates of the gold particles were assigned using Image $J$ and were used to calculate the co-localization between the two populations of gold. Smaller black dots indicate 2-nm gold labeling RFP; larger red dots indicate 6-nm gold labeling GFP. C The extent of co-localization, $L_{\text {biv }}(r)-r$, was plotted against the length scale, $r$, in nanometer. $L_{\text {biv }}(r)-r$ values above the $95 \%$ confidence interval $(95 \% \mathrm{CI})$ indicate statistically meaningful co-localization

$$
\begin{aligned}
& K_{\mathrm{bs}}(r)=\frac{A}{n_{\mathrm{b}} n_{\mathrm{s}}} \sum_{i=1}^{n_{\mathrm{b}}} \sum_{j=1}^{n_{\mathrm{s}}} w_{i j} 1\left(\left\|x_{i}-x_{j}\right\| \leq r\right) \\
& K_{\mathrm{sb}}(r)=\frac{A}{n_{\mathrm{b}} n_{\mathrm{s}}} \sum_{i=1}^{n_{\mathrm{s}}} \sum_{j=1}^{n_{\mathrm{b}}} w_{i j} 1\left(\left\|x_{i}-x_{j}\right\| \leq r\right) \\
& L_{\mathrm{biv}}(r)-r=\sqrt{\frac{K_{\mathrm{biv}}(r)}{\pi}}-r
\end{aligned}
$$

where $K_{\mathrm{bs}}(r)$ depicts the distribution of the 6-nm ("b" means big) gold particles with respect to each 2-nm ("s" means small) gold particle and reciprocally $K_{\mathrm{sb}}(r)$ characterizes the distribution of small gold particles with respect to each big gold particle. $K_{\text {biv }}(r)$ estimator combines the both $K_{\mathrm{bs}}(r)$ and $K_{\mathrm{sb}}(r)$ to generate a single function, where $n_{\mathrm{b}}$ means number of big (6-nm) gold particles and $n_{\mathrm{s}}$ means number of small (2-nm) gold particles 
and other notation is as in Eq. 1 and 2 (Fig. 2C). Monte Carlo simulations then yield a 95\% confidence interval, which further standardizes $L_{\text {biv }}(r)-r$. We then use a term, LBI, as a defined integral of the standardized $L_{\mathrm{biv}}(r)-r$ function to summarize statistic of extent of co-localization (Zhou et al. 2014):

$$
L B I=\int_{10}^{110} \operatorname{Std} L_{\mathrm{biv}}(r)-r \cdot \mathrm{d} r
$$

For each condition, a minimum of 15-20 PM sheets are imaged and analyzed. Bootstrap tests used in the univariate analysis are also used to evaluate the statistical significance of differences between two bivariate data sets.

\section{DISCUSSION}

The spatial distribution of the membrane proteins and the lipids on/in the PM is critical to their activities and cell signaling/function. Many speculate heterogeneity in the PM, where the proteins and the lipids laterally segregate into specialized domains, which play essential roles in the function of these proteins, i.e., their ability to be recruited to the activation sites and be activated, their ability to recruit downstream effectors, and their ability to establish signaling network with other components in the PM (Hancock 2003, 2006; Nan et al. 2013; Simons and Toomre 2000; Zhou and Hancock 2015). The traditional fluorescence microscopy can qualitatively pinpoint the location of excess localization, such as the observation of puncta formation in cells. However, these techniques do not have high enough spatial resolution to yield quantitative measurement of the extent of the lateral segregation because of the optical resolution limit of $200 \mathrm{~nm}$. FRET-based techniques are powerful methodologies in measuring dimerization/oligomerization within a distance less than $10 \mathrm{~nm}$ (Meyer et al. 2006). The effective distance is limited to $10 \mathrm{~nm}$ because the fluorescence energy transfer only occurs when the donors and the acceptors are less than $10 \mathrm{~nm}$ apart. Additionally, traditional techniques for measuring membrane recruitment, such as immuno-blotting, cannot distinguish between PM fractions versus endomembrane fractions.

The EM-spatial mapping, on the other hand, is a highly quantitative analysis to mathematically characterize the ability of the membrane proteins or the lipids to laterally distribute on the PM. This technique has a length scale from 5 to $240 \mathrm{~nm}$, providing crucial information on the nano-scale spatial organization of the PM components over a long-distance range. The quantitative parameters, such as clustered fraction and number of proteins per cluster, yield critical information on the extent of oligomerization and the potential role of oligomers in signal transmission. Because our protocol is optimized to attached intact PM sheets, but not other endomembrane compartments, to the EM grids, the EMspatial analysis effectively quantifies the spatial distribution of the membrane proteins exclusively on the intact PM, without the background from endomembranes. The bivariate co-clustering analysis mathematically examines the ability of the two populations of species to colocalize on the PM, as well as substrate-effector recruitment on the PM. This co-localization methodology characterizes oligomerization on a length scale $(8-240 \mathrm{~nm})$ that cannot be obtained via FRET-based imaging methods, as the energy transfer between FRET donor and acceptor is limited to $<10 \mathrm{~nm}$. We have used the bivariate analysis extensively to map the lipid composition of various Ras nanoclusters.

The primary weakness of the EM-spatial mapping methodology is that we need to flatten and fix cells to attach the apical/basolateral PM onto the EM grids for imaging. Although the results obtained via the EM-spatial mapping technique have been validated by traditional methods using intact/live cells, such as FLIM-FRET, FRAP, FCS, and single particle tracking, and molecular simulations from our group and other investigators, the EM technique still does not reflect the most native state of cells. The EM-spatial mapping technique also relies on the use of fluorescently tagged proteins. It is still not clear how tagging a GFP molecule could potentially influence the lateral movement/ distribution of a protein. Thus, it is critical to validate that the fluorescent tag does not influence the function of the proteins tested. The reliance of the EM-spatial analysis on the over-expression of GFP-tagged proteins/ peptides also presents issues because an increase in the protein level could potentially influence the extent of nanoclustering of the proteins on the PM. Interestingly, early studies have shown convincing evidence that membrane proteins, especially Ras small GTPases, maintain constant clustered fraction and the extent of 
the nanoclustering over many orders of magnitude of the expression level (Tian et al. 2007).

When mapping the lipid distribution in the PM, we mainly use the GFP-tagged lipid-binding domains, such as the well-established $\mathrm{C} 2$ and PH domains, to track the location of lipids in the PM. However, the overexpression of the lipid-binding domains could potentially influence cell signaling by competitive binding to the lipids, thus effectively quenching the available concentrations of these lipids. This could especially become problematic when studying the lipid co-localization with the proteins that have the same lipid-binding domains. Thus, when studying the lipid composition of the membrane proteins, proper control experiments should be performed. For instance, activities of the involved signaling cascades should be checked with/without the over-expression of certain lipid-binding domain to ensure that the over-expressed lipid-binding domain does not artificially influence the localization and the activities of the membrane proteins by competing with the proteins for any lipid. The co-localization between the lipids and the membrane protein should also be validated using the fluorescent lipids in a FRET-based technique.

Detailed information of the reagents used is listed in Table 3.

Table 3 Reagents utilized in this work

\begin{tabular}{lll}
\hline Reagent & Company & Cat\# \\
\hline Pioloform & TED PELLA & 19244 \\
Choroform & Electron Microscopy & 12550 \\
Sciences (EMS) & \\
Poly-L-lysine & Sigma & P8920-100ML \\
EM copper grids & EMS & G200-CU \\
Trisodium citrate & Sigma & C3674-100G \\
Tannic acid & EMS & 21710 \\
Potassium carbonate & Sigma & P5833-500G \\
Potassium acetate & Sigma & P1190-1KG \\
Glycerol & Sigma & G55-1L \\
Paraformaldehyde & EMS & 1570 \\
Glutaraldehyde & EMS & 16220 \\
Fish gelatin & Sigma & G7765-250ML \\
BSA & Sigma & A6003-100G \\
Gold chloride & Sigma & $254169-5 G$ \\
\hline
\end{tabular}

Acknowledgements This work was supported by the Cancer Research and Prevention Institute of Texas (CPRIT) (RP130059 and RP170233) and the NIH (P30 DK 56338).

\section{Compliance with Ethical Standards}

Conflict of interest Yong Zhou and John F. Hancock declare that they have no conflict of interest.

Human and animal rights and informed consent This article does not contain any studies with human or animal subjects performed by any of the authors.

Open Access This article is distributed under the terms of the Creative Commons Attribution 4.0 International License (http:// creativecommons.org/licenses/by/4.0/), which permits unrestricted use, distribution, and reproduction in any medium, provided you give appropriate credit to the original author(s) and the source, provide a link to the Creative Commons license, and indicate if changes were made.

\section{References}

Baumgart T, Hess ST, Webb WW (2003) Imaging coexisting fluid domains in biomembrane models coupling curvature and line tension. Nature 425:821-824

Diggle PJ, Mateu J, Clough HE (2000) A comparison between parametric and non-parametric approaches to the analysis of replicated spatial point patterns. Adv Appl Probab $32: 331-343$

Hancock JF (2003) Ras proteins: different signals from different locations. Nat Rev Mol Cell Biol 4:373-384

Hancock JF (2006) Lipid rafts: contentious only from simplistic standpoints. Nat Rev Mol Cell Biol 7:456-462

Levental I, Grzybek M, Simons K (2011) Raft domains of variable properties and compositions in plasma membrane vesicles. Proc Natl Acad Sci USA 108:11411-11416

Meyer BH, Segura JM, Martinez KL, Hovius R, George N, Johnsson $\mathrm{K}$, Vogel H (2006) FRET imaging reveals that functional neurokinin-1 receptors are monomeric and reside in membrane microdomains of live cells. Proc Natl Acad Sci USA 103:2138-2143

Nan X, Collisson EA, Lewis S, Huang J, Tamguney TM, Liphardt JT, McCormick F, Gray JW, Chu S (2013) Single-molecule superresolution imaging allows quantitative analysis of RAF multimer formation and signaling. Proc Natl Acad Sci USA 110:18519-18524

Plowman SJ, Muncke C, Parton RG, Hancock JF (2005) H-ras, K-ras, and inner plasma membrane raft proteins operate in nanoclusters with differential dependence on the actin cytoskeleton. Proc Natl Acad Sci USA 102:15500-15505

Prior IA, Muncke C, Parton RG, Hancock JF (2003a) Direct visualization of Ras proteins in spatially distinct cell surface microdomains. J Cell Biol 160:165-170

Prior IA, Parton RG, Hancock JF (2003b) Observing cell surface signaling domains using electron microscopy. Sci STKE, PL9

Ripley BD (1977) Modeling spatial patterns. J R Stat Soc Series B Stat Methodol 39:172-192

Simons K, Toomre D (2000) Lipid rafts and signal transduction. Nat Rev Mol Cell Biol 1:31-39 
Tian T, Harding A, Inder K, Plowman S, Parton RG, Hancock JF (2007) Plasma membrane nanoswitches generate highfidelity Ras signal transduction. Nat Cell Biol 9:905-914

Veatch SL, Keller SL (2002) Organization in lipid membranes containing cholesterol. Phys Rev Lett 89:268101

Zhou Y, Hancock JF (2015) Ras nanoclusters: versatile lipid-based signaling platforms. Biochim Biophys Acta 1853:841-849

Zhou Y, Cho KJ, Plowman SJ, Hancock JF (2012) Nonsteroidal antiinflammatory drugs alter the spatiotemporal organization of Ras proteins on the plasma membrane. J Biol Chem 287:16586-16595
Zhou Y, Liang H, Rodkey T, Ariotti N, Parton RG, Hancock JF (2014) Signal integration by lipid-mediated spatial cross talk between Ras nanoclusters. Mol Cell Biol 34:862-876

Zhou Y, Wong CO, Cho KJ, van der Hoeven D, Liang H, Thakur DP, Luo J, Babic M, Zinsmaier KE, Zhu MX, Hu HZ, Venkalachalam K, Hancock JF (2015) Signal transduction. Membrane potential modulates plasma membrane phospholipid dynamics and K-Ras signaling. Science 349:873-876

Zhou Y, Prakash P, Liang H, Cho KJ, Gorfe AA, Hancock JF (2017) Lipid-sorting specificity encoded in K-Ras membrane anchor regulates signal output. Cell 168(239-251):e216 